\title{
Los Objetos Virtuales de Aprendizaje Transdisciplinarios, como Alternativa a la Problemática Enseñanza y Aprendizaje de la Física en la Modalidad Virtual
}

\section{Transdisciplinary Virtual Learning Objects, as an Alternative to the Problematic Teaching and Learning of Physics in the Virtual Modality}

José Antonio Aceituno-Mederos ${ }^{1}$, María Elena Córdoba², María Encarnación Acosta-Hernández ${ }^{3}$, Manuel De J. Reyes-Guzmán 4 y Ricardo J. Morales De Jesús ${ }^{5}$

\section{() \\ EDICIÓN: @e-CIVTAC}

Recibido: 14/julio/2020

Aceptado: 5/agosto/2020

Publicado: 25/septiembre/2020

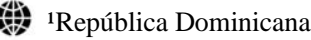
${ }^{2}$ República Dominicana

${ }^{3}$ República Dominicana

${ }^{4}$ Puerto Rico

${ }^{5}$ Puerto Rico

\section{IIIIInstitución}

${ }^{1}$ Instituto Tecnológico de Santo Domingo 2Instituto Tecnológico de Santo Domingo ${ }^{3}$ Instituto Tecnológico de Santo Domingo ${ }^{4}$ Universidad de Puerto Rico

${ }^{5}$ Universidad de Puerto Rico

\section{Correo Eletrónico}

1jose.aceituno@intec.edu.do ${ }^{2}$ maría.cordoba@intec.edu.do 3 maría.acosta@intec.edu.do ${ }^{4}$ manuel.reyes3@upr.edu ${ }^{5}$ ricardo.morales1@upr.edu

\section{ORCID}

'https://orcid.org/0000-0001-7597-7850 ${ }^{2 h t t p s: / / o r c i d . o r g / 0000-0002-8827-457 X ~}$ ${ }^{3}$ https://orcid.org/0000-0003-2164-262X ${ }^{4}$ https://orcid.org/0000-0001-9491-3641 ${ }^{5} \mathrm{https} / / /$ orcid.org/0000-0002-9738-5546

\section{Citar así: CCAPA / IEEE}

Aceituno-Mederos, J., Córdoba, M., AcostaHernández, M., Reyes-Guzmán, M. \& Morales De Jesús, R. (2020). Los Objetos Virtuales de Aprendizaje Transdisciplinarios, como Alternativa a la Problemática Enseñanza y Aprendizaje de la Física en la Modalidad Virtual. Revista TecnológicaEducativa Docentes 2.0, 9(2), 119-131.

J. Aceituno-Mederos, M. Córdoba, M. Acosta-Hernández, M. Reyes-Guzmán y R. Morales De Jesús, "Los Objetos Virtuales de Aprendizaje Transdisciplinarios, como Alternativa a la Problemática Enseñanza y Aprendizaje de la Física en la Modalidad Virtual", RTED, vol. 9, n. ${ }^{\circ}$ 2, pp. 119-131, sep. 2020.

\section{Resumen}

La inclusión de la enseñanza virtual en las universidades es un hecho inaplazable, que requiere un soporte investigativo capaz de garantizar ofertas académicas de calidad que den respuestas a los requerimientos del mundo actual, a las expectativas de la sociedad en general y de los estudiantes en particular. En el Instituto Tecnológico de Santo Domingo (INTEC) a partir de un curso de Física Mecánica Virtual se investigó por un periodo de más de dos años para identificar los obstáculos vinculados con el aprendizaje de la Física virtual desde la perspectiva de los actores directos involucrados en el problema (estudiantes y docentes). El diseño fue cuasi experimental, la recolección de datos se realizó con un examen estandarizado tanto para pre como post prueba, así como instrumentos de recogida de datos diseñados para este estudio, los cuales fueron validados mediante consulta con expertos y aplicación de pruebas piloto. Los resultados obtenidos por los estudiantes en modalidad virtual fueron comparados con los obtenidos previamente por estudiantes que recibieron la asignatura de forma presencial. Entre los criterios de análisis se consideró el perfil de los usuarios del curso virtual, desempeño según la calificación obtenida, estructura del curso, opiniones sobre ventajas que percibieron, dificultades y cambios que sugieren para mejorar versiones futuras. Se concluyó que las modalidades presencial y virtual del curso de Física son esencialmente similares; a pesar de ello hay una cierta preferencia por la enseñanza presencial. Todo lo cual derivó en la propuesta de construir Objetos Virtuales de Aprendizaje transdisciplinarios (OVAt) como posible solución a las carencias encontradas.

Palabras clave: Física virtual, transdisciplinariedad, objetos virtuales de aprendizaje.

\section{Abstract}

The inclusion of virtual teaching in universities is an unavoidable fact, which requires research support capable of confirming quality academic offerings that respond to the requirements of today's world, the expectations of society in general and of students. At the Technological Institute of Santo Domingo (INTEC), from a course of Virtual Mechanical Physics, research was carried out for a period of more than two years to identify the Obstacles linked to learning virtual physics from the perspective of the direct actors involved in the problem (students and teachers). The design was quasi-experimental, data collection was performed with a standardized examination for both pre and posttest, as well as the data collection instruments carried out for this study, which were validated through consultation with experts and application of pilot tests. The results obtained by the students in the virtual modality were compared with those previously obtained by the students who received the subject in person. The analysis criteria will consider the profile of the users of the virtual course, performance according to the grade obtained, course structure, opinions on perceived benefits, difficulties and changes that may improve future versions. It was concluded that the face-to-face and virtual modalities of the Physics course are essentially similar; Despite this, there is a certain preference for face-to-face teaching. All of which was derived in the proposal to build transdisciplinary Virtual Learning Objects (OVAt) as a possible solution to the deficiencies found.

Keywords: Virtual Physics, transdisciplinary, virtual learning objects. 
Los Objetos Virtuales de Aprendizaje Transdisciplinarios, como Alternativa a la Problemática Enseñanza y Aprendizaje de la Física en la Modalidad Virtual.

\section{Introducción}

La experiencia de varios años impartiendo docencia en el área de ciencias básicas, ofreció a un docente de Física el escenario propicio para recopilar información de un proceso en el que los estudiantes llegan con temor y desmotivados con esta asignatura. El intento de múltiples estrategias para lograr motivar y que no siempre resultaban atinadas, sumado a quejas de docentes durante años respecto de diversas dificultades presentadas por los estudiantes para aprender matemática, física, o química, resultaron el motor para investigar de manera profunda en un intento de solución a este tema.

Los estudiantes que ingresaban a la asignatura de Física parecían no tener el menor interés en ella y a pesar de intentar múltiples estrategias, no se lograba encontrar una modalidad significativamente diferente. Enseñar física en muchos países resulta de manera uniforme un dolor de cabeza desde el punto de vista pedagógico, por esta razón se han desarrollado, a nivel mundial, multitud de programas, frecuentemente más atractivos para los docentes que para los estudiantes. Estos interesantes programas y estrategias variadas para aplicarse en formato presencial y virtual no parecen significar un gran salto en la motivación de los estudiantes ni en los logros de aprendizaje, comparado con lo que antes se tenía.

El ofrecimiento de algunas sesiones de física en modalidad virtual fue una oportunidad para un estudio comparativo en cuanto a si los estudiantes, del mismo docente en ambas modalidades, presentaban diferentes niveles de desempeño, dependiendo de la modalidad. La enseñanza en modalidad virtual tuvo como premisa fomentar el trabajo independiente y cooperativo, en el entendido de que la educación a distancia es un sistema ecológico con reglas muy diferentes del sistema educativo tradicional.

El contexto tecnológico digital provee retos conjuntamente con oportunidades a todos los actores de esta modalidad que aún no se han desentrañado en su totalidad En la comparación que se realizó de estudiantes de física en modalidad presencial y virtual con el mismo docente, no se encontraron diferencias significativas en desempeño, esta investigación se aprovechó para recabar opiniones de estudiantes y docentes sobre cómo incorporar estrategias de mejoras al proceso de enseñanza-aprendizaje.

El análisis de los datos obtenidos en la comparación realizada dejó expuesta la necesidad de incorporar estrategias novedosas para el aprendizaje, que resultarán cualitativamente diferentes a lo que se había hecho hasta la fecha. Esta fue la razón por la cual se pensó en imprimir a esta propuesta una visión transdisciplinaria de la educación, e integrando a profesionales de diferentes disciplinas. De esta manera se creó la idea de construir Objetos Virtuales de Aprendizaje (OVA) con elementos transdisciplinares (OVAt)

Construir OVAt alineados con diversas características detectadas o sugeridas por los afectados directos por el problema, fue el objetivo principal de este trabajo. Los estudiantes participantes definieron los componentes fundamentales de cualquier experiencia virtual que les resulte significativa. Construir este OVAt es tan solo el inicio de una metodología de creación de paquetes de programas utilizables en principio en cualquier disciplina. Es a su vez una invitación a todo docente para inscribirse en una concepción transdisciplinaria de la educación.

El diseño de los OVAt tiene como premisa que toda educación a distancia puede equilibrar relaciones tecnológicas entre profesores y estudiantes, pues cada cual queda a expensas de sus capacidades, competencias personales, nuevas formas de colaboración, aprendizaje individualizado y autodirección. El diseño considera además el acceso y uso apropiado de información como prerrequisito indispensable de una educación a distancia de calidad. También ofrece a cada participante oportunidades de exponenciar o coartar su avance intelectual estudiando a distancia.

La experiencia tecnológica de educación a distancia en la caribeña muestra desarrollo y características particulares, tanto en el caso de República Dominicana como en el de Puerto Rico. Comenzó de manera muy gradual en algunos casos mientras que en otros casos era inexistente; sin embargo, nuestra sociedad muestra una clara disposición para incorporar nuevas tecnologías de comunicación e información en sus vidas, en medida de sus posibilidades, aun en detrimento de una buena educación, esto tal vez motivado por situaciones de crisis tales como huracanes, terremotos o pandemias.

De acuerdo con nuestra interpretación del trabajo de Mujica (2019), la educación virtual debe ser accesible y acorde a las demandas de las nuevas generaciones a nivel global, además de integrar las tecnologías de la información. 
Esto junto al desarrollo de las competencias humanas generales brindan un excelente momento para aunar esfuerzos donde se integren dichas tecnologías con las capacidades de administradores, docentes y estudiantes, graduados y subgraduados para diseñar ambientes de enseñanza/aprendizajes conducentes al éxito académico en la educación a distancia.

Por otra parte, los estudiantes en su mayoría encuentran difíciles y aburridos los problemas conceptuales y numéricos icónicos de las ciencias físicas, porque no encuentran aplicación directa con la vida cotidiana, ni relación entre los problemas mundiales que acaparan los noticieros con los fundamentos de la ciencia y la tecnología que se enseñan en la universidad. La falta de interés en los fundamentos epistemológicos de la ciencia y su desarrollo histórico podrían ser un indicador de la falta de motivación que los estudiantes sienten ante los retos educativos que enfrentan. La motivación juega un papel crucial en los procesos de aprendizaje (Pintrich 1999, Lieury \& Fenouillet 2016) y es de naturaleza emocional por lo que el estado afectivo influye en el proceso de cognición (Gable \& HarmonJones, 2010).

La investigación presentada intenta aplicar un enfoque transdisciplinario para lograr motivar, empoderar al estudiante para lograr aprendizajes significativos, y de esta forma crear una educación vigente, perdurable, con impacto positivo en su vida y comunidad. Los elementos estéticos y lúdicos esenciales al proceso se proponen como motor motivacional. La importancia de la motivación para mantener disciplina y lograr el cumplimiento de responsabilidades, expuesto por autores como Escobar (2017). En nuestra propuesta se vincula el uso de refuerzos positivos, en el contexto de la teoría del condicionamiento operante de Skinner, con la incorporación de miríadas de estímulos audiovisuales para fomentar una participación más activa en los medios virtuales.

El colectivo de investigadores, cada uno desde sus disciplinas y fortalezas, propone un acercamiento transdisciplinario para crear materiales motivadores para una educación virtual inclusiva y accesible a todos. Inicialmente se identificaron dimensiones vinculadas a esta problemática en cuestión, permitiendo a los proponentes y sus colaboradores, entre los que se encuentran los afectados directos en el problema, realizar actividades académicas apropiadas para atender aspectos disciplinares de la educación a distancia, y aspectos que van más allá de las fronteras disciplinares, logrando integración disciplinar, por un lado, así como trascender los límites, creando una experiencia de aprendizaje significativo y duradero. El Instituto Tecnológico de Santo Domingo (INTEC) ya ha pasado por los primeros estadios de virtualización a nivel de unidad y su experiencia es muy útil para crear materiales aplicables a escala regional que permitan atender problemas académicos compartidos.

\section{Metodología}

La metodología utilizada en esta investigación implicó un proceso en dos etapas, una primera correspondiente al análisis comparativo de resultados de estudiantes de física que tomaron la asignatura en modalidad virtual respecto a quienes la tomaron en forma presencial. Además, en esta etapa se identificaron con la colaboración de estudiantes y docentes, las problemáticas más sentidas alrededor del aprendizaje de esta asignatura. Una segunda etapa se deriva de la anterior e involucra un equipo de investigadores de diferentes disciplinas a fin de diseñar los Objetos Virtuales de Aprendizaje enriquecidos con una metodología transdisciplinaria (OVAt)

La primera etapa de esta investigación se desarrolló con elementos combinados del abordaje mixto cuantitativo y cualitativo. El diseño fue cuasi experimental utilizando los grupos de estudiantes inscritos en la asignatura de Física, a los cuales se les aplicó como pre y post prueba un examen estandarizado que fue validado previamente y además encuestas para la recolección de los criterios de los estudiantes.

El propósito fundamental de este estudio es atender las diversas problemáticas identificadas en el aprendizaje al implementar la enseñanza virtual de las Ciencias Físicas, con una propuesta que resulte cualitativamente diferente a las que se han desarrollado hasta la fecha. Como insumos para este diseño se utilizará la información sobre los obstáculos identificados previamente, vinculados con el aprendizaje de la Física virtual según los actores directos involucrados en el problema (estudiantes y docentes), siendo esta la base para la construcción de un diseño instruccional para la creación de OVAt. 


\section{Participantes}

Para llevar a cabo este trabajo han intervenido diversos actores según las etapas. Para el presente estudio la muestra se compone de 360 estudiantes, en una distribución aproximada de 40 por cada sección por trimestre. Los docentes a quienes se les consultó sobre las principales dificultades que percibían en los estudiantes para el aprendizaje de la Física, ascienden a ocho. Los criterios de selección para el estudio más reciente consistieron en que fueran estudiantes activos de la asignatura de Física en modalidad virtual, y en el caso de los estudiantes en modalidad presencial, que tomaran la clase con el mismo docente.

\section{Instrumentos de Recolección de Datos}

Se utilizaron exámenes escritos y encuestas para la recolección de los datos. Los exámenes escritos para evaluar aprendizajes de Cinemática fueron la prueba estandarizada: "Testing student interpretation of kinematicsgraph". La encuesta se elaboró a partir de la propuesta "Cuestionario de evaluación de la calidad de los cursos virtuales de la UNED", y fue adaptada a los propósitos del estudio por los autores. Este instrumento evalúa tres dimensiones fundamentales; calidad del entorno donde se virtualiza el curso, calidad de la metodología didáctica utilizada en el curso y calidad técnica del curso. Para el procesamiento estadístico de la data experimental, se utilizaron los programas Microsoft Excel y R.

\section{Procedimientos}

El estudio describe el comportamiento de las variables que se midieron durante los ocho trimestres estudiados, su diseño fue no experimental longitudinal donde se levantó data durante varios trimestres consecutivos. Al inicio del curso se explicaba a los estudiantes que se estarían levantando sus opiniones para una investigación con el propósito de mejorar la calidad de la enseñanza de la Física, a fin de lograr mejores aprendizajes, por lo que cualquier dato que pudieran aportar, adicional a los que se les solicitaban durante el trimestre, sería de gran utilidad. La encuesta fue aplicada cada trimestre y se levantaron datos de comentarios, propuestas y sugerencias durante todo el trimestre en un diario de de clases.

Este proyecto de investigación tuvo una duración total de ocho trimestres consecutivos. Además, se compara con datos de estudiantes presenciales acumulados por un periodo de tres trimestres, lapso durante el cual coincidió impartiendo docencia el mismo docente con clases en ambas modalidades.

\section{Resultados}

Como parte de su plan de desarrollo, el INTEC tiene como meta ampliar la oferta académica actual a otras modalidades. Para esto ha trabajado en los pasados años en el diseño de asignaturas para el ofrecimiento de modo virtual y de formas híbridas utilizando acompañamiento del Aula Virtual. Para el actual estudio se utilizaron los estudiantes matriculados en el curso de Física Virtual I, impartido por primera vez en INTEC en el trimestre Mayo Julio de 2017. En esa ocasión se inscribieron en el curso un total de 40 estudiantes. El rendimiento, medido a partir de notas obtenidas en el curso, se recoge en la Tabla \#1 de los apéndices y se presenta resumido en la gráfica 1.

Se observa un $30 \%$ de los estudiantes matriculados retirando la asignatura antes de tomar el examen final, un $22.5 \%$ no aprobó y un $47.55 \%$ aprobó. El análisis final de calificaciones de los estudiantes promovidos muestra un $22.5 \%$ obteniendo buena calificación (A o B), mientras el $25 \%$ muestra un rendimiento regular con calificación de C.

Estos resultados son similares a la media histórica de calificaciones del curso Física I en modalidad presencial en INTEC donde alrededor del $50 \%$ de la matrícula total aprueban esta asignatura.

\section{Gráfica 1}

Evaluaciones 2017.

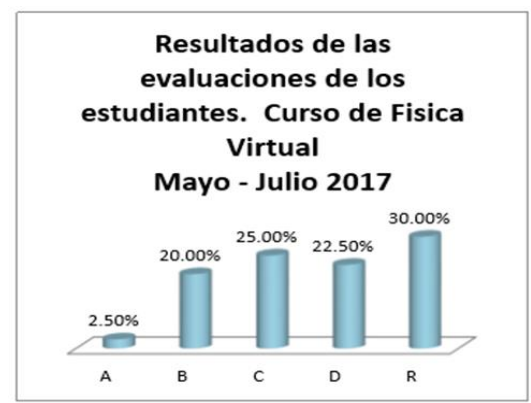

Nota. Evaluaciones. Curso de Física Virtual, elaboración propia (2017). 
Como parte del estudio se ofreció un cuestionario de satisfacción donde los estudiantes expresaron aspectos positivos, negativos y los cambios sugeridos para el mejorar el Curso de Física Virtual I. Estos datos se encuentran organizados en la Tabla \# 2 de los apéndices, los cuales se grafican y se discuten a continuación.

\section{Gráfico 2}

Opiniones de los estudiantes.

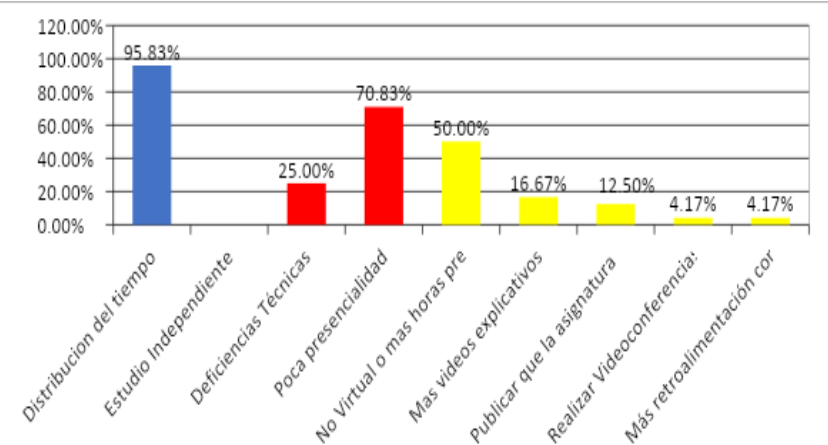

Nota. Opiniones de los estudiantes, elaboración propia (2017).

El 95.83\% de los estudiantes opinaron que el curso les permitía usar mejor su tiempo y los preparaba para aprender por sí solos. Sin embargo, el $25 \%$ reportó algún tipo de dificultad técnicas con Moodle (plataforma utilizada para ofrecer el curso), esto en su opinión les afectó el aprovechamiento.

Un resultado interesante de la encuesta es que los estudiantes demandaban más contacto presencial con el profesor, lo que resulta cónsono con las ideas de Barráez (2020) sobre la importancia de la interacción sincrónica cotidiana como componente fundamental para que la transformación a medios virtuales sea efectiva y positiva. Este aspecto llama la atención, ya que es una asignatura impartida en modalidad virtual, donde tradicionalmente se espera menos intervención del docente.

Cuando analizamos las propuestas de cambios al curso hechas por los estudiantes, vemos el $50 \%$ opinando que la asignatura no debe impartirse en esta modalidad, también indican que debe haber una mayor cantidad de videos tutoriales explicando cómo resolver ejercicios similares a los asignados. Indican que los tutoriales deben incluir algún tipo de retroalimentación en caso de ser contestados equivocadamente.

Los resultados del cuestionario nos llevan a reflexionar acerca de que nuestros estudiantes no están satisfechos con el nivel de preparación académica alcanzada al tomar la asignatura de Física Mecánica en modalidad completamente virtual. Esta percepción estudiantil negativa no debe significar el abandono de la educación virtual, sino un reenfoque para incluir acciones afirmativas de solución a todas estas dificultades.

A partir de los resultados obtenidos, se trabajó en una versión mejorada del Curso de Física Virtual I que se impartió en el trimestre agosto-octubre de 2018 en INTEC. En esta ocasión se realizaron pruebas de entrada y salida a los estudiantes matriculados en el tema de Cinemática usando exámenes estandarizados creados para estos fines por Beichner (1994). Las evaluaciones se hicieron al comenzar a estudiar el tema en cuestión y al concluir dicho tema. La Tabla \#3 del apéndice organiza la data obtenida y se presenta resumida en el siguiente gráfico:

\section{Gráfico 3}

Resultados INTEC 2018.

\section{Cinemática INTEC}

\section{Agosto-Octubre 2018}

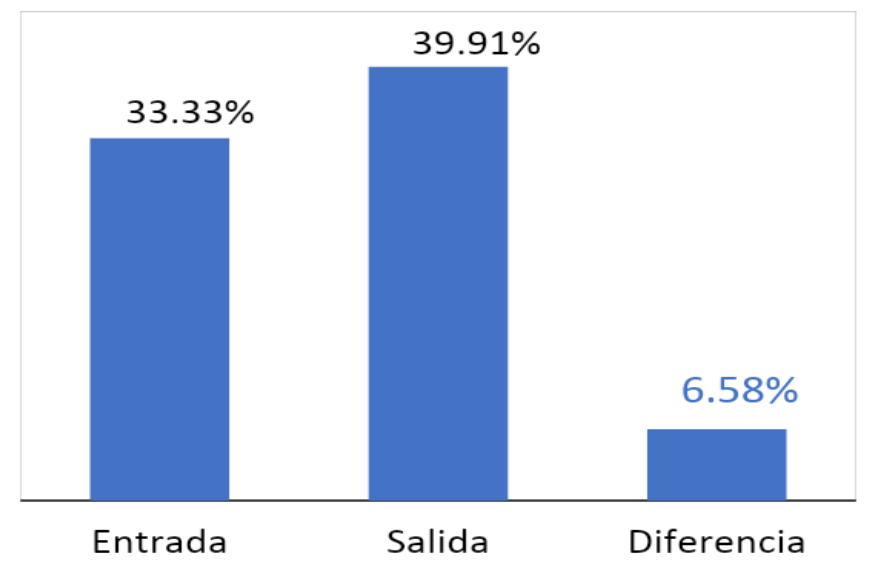

Nota. Resultados de evaluaciones en INTEC 2018 elaboración propia (2017).

Se evidencia un aumento promedio de $6.58 \%$ en el rendimiento de los estudiantes después de haber estudiado el tema usando el curso de Física Virtual I. Consideramos que este aumento en rendimiento es bajo y se debe aspirar a que el rendimiento de salida esté cerca de un $80 \%$, sin embargo, esto es independiente de la modalidad utilizada (virtual o presencial).

Al comparar los resultados de estos exámenes estandarizados con los obtenidos por otros estudiantes en el pasado en cursos presenciales en INTEC vemos que el perfil es muy similar. 
Los Objetos Virtuales de Aprendizaje

Transdisciplinarios, como Alternativa a la Problemática

Enseñanza y Aprendizaje de la Física en la Modalidad Virtual.

Estas evaluaciones se hicieron en los momentos de antes y después de estudiar el tema de Cinemática en la modalidad presencial y los resultados lo podemos ver en las siguientes gráficas:

\section{Gráfica 4}

Resultados INTEC 2011.

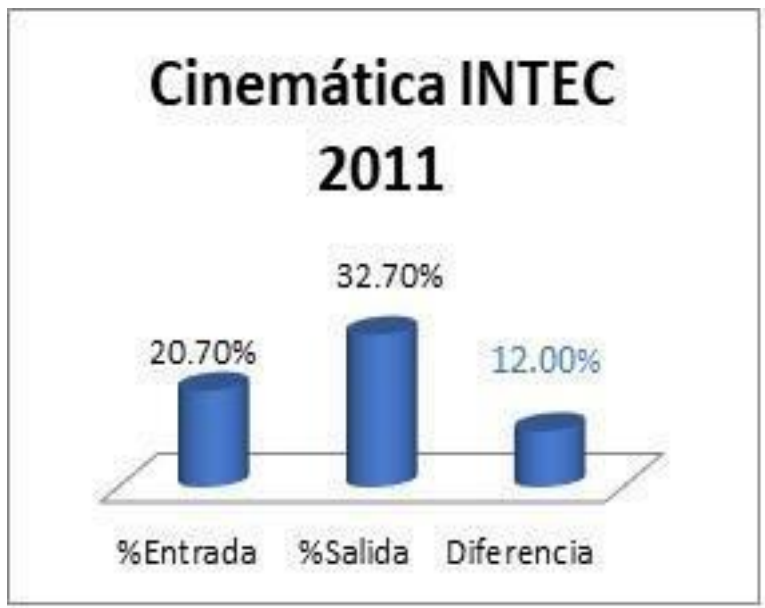

Nota. Resultados de evaluaciones en INTEC 2011, elaboración propia.

\section{Gráfica 5}

Resultados INTEC 2015.

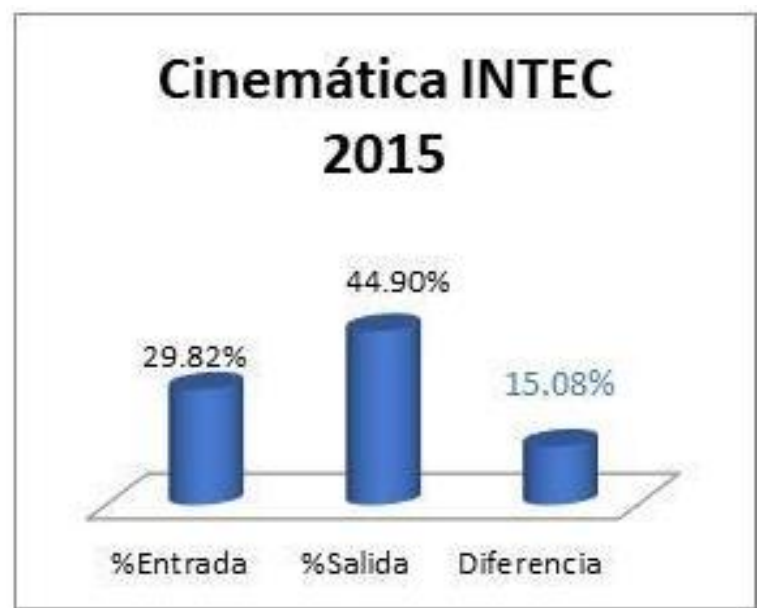

Nota. Resultados de evaluaciones en INTEC 2015, elaboración propia.

Estas gráficas nos evidencian los cambios en el nivel de conocimientos de cinemática de los estudiantes que reciben la asignatura, independientemente de la modalidad, son positivos, pero mucho menor de lo deseable, pues en el mejor de los casos se logra solo un $49.9 \%$ de respuestas correctas. Al comparar notamos que no hay diferencias aparentes entre recibir la Física Mecánica en la modalidad virtual o presencial.
Para sustentar este planteamiento se hizo un estudio con dos secciones de clases en el trimestre noviembre 2018 - enero 2019. El grupo control recibió la Física Mecánica I en modalidad presencial y el grupo experimental recibió la asignatura en modalidad virtual. Estos datos se recogen en las Tablas 4 y 5 del apéndice.

Los datos utilizados son las notas obtenidas por los estudiantes al realizar la prueba estandarizada de cinemática, que se ha venido aplicando a lo largo de este estudio, antes y después de recibir el tema en la modalidad correspondiente.

¿Qué notas medias de entrada y salida tienen los grupos virtual y presencial?

\section{Gráfico 6}

Entrada Presencial.

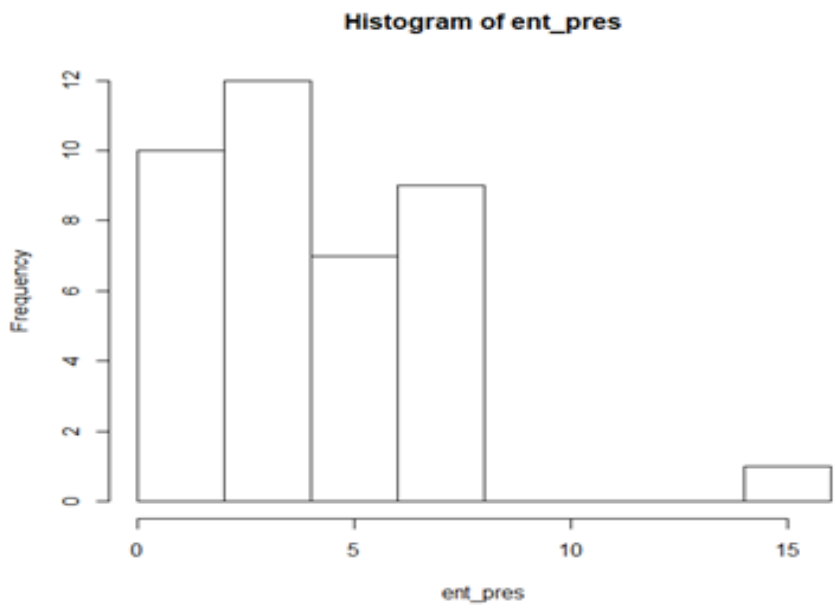

Nota. Entrada Presencial: 4.564103, elaboración propia.

\section{Gráfico 7}

Entrada Virtual.

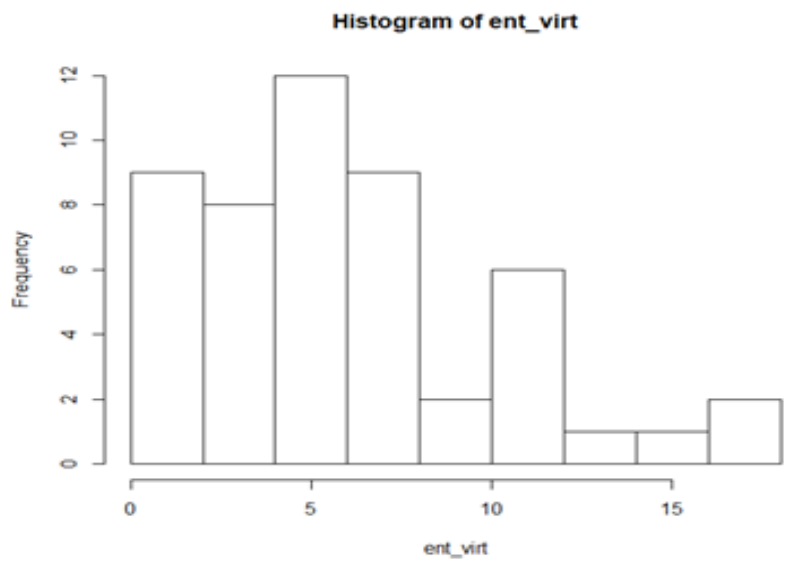

Nota. Entrada Virtual 6.48, elaboración propia. 


\section{Gráfico 8}

\section{Salida Presencial.}

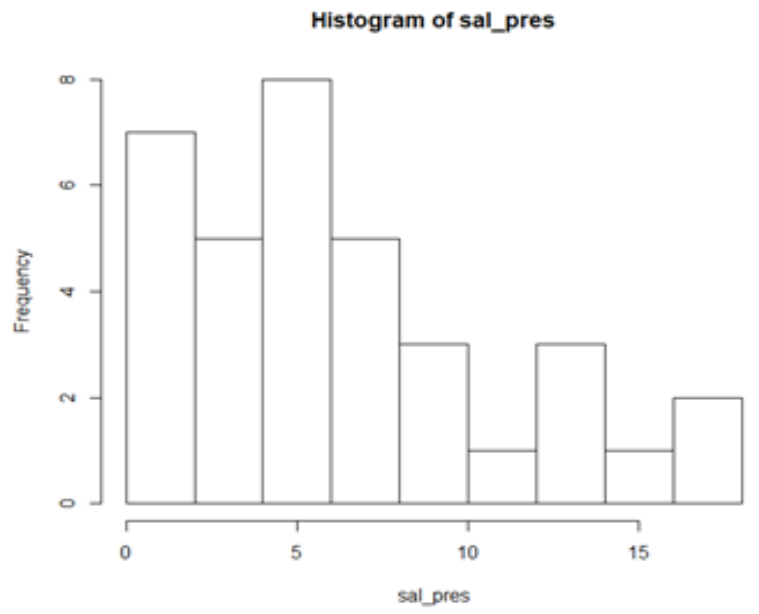

Nota. Salida Presencial: 6.971429, elaboración propia.

\section{Gráfico 9}

Salida Virtual.

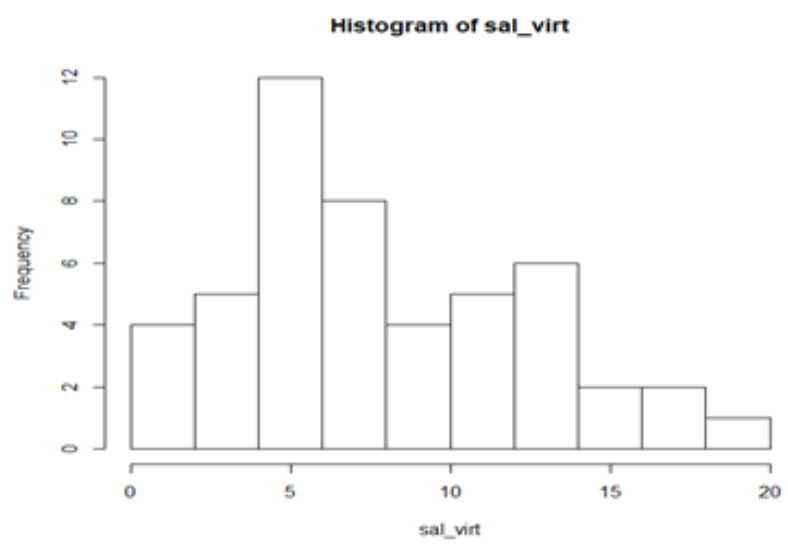

Nota. Salida Virtual: 8.244898, elaboración propia.

Vemos que la nota media de salida es más alta en ambos grupos y, a su vez, que las notas de entrada y salida de los estudiantes son más altas en el grupo virtual. Debe aclararse que en el cálculo de estas notas medias se están excluyendo los estudiantes no presentados a cada uno de los exámenes. Hay estudiantes que se presentaron al examen de entrada y no al de salida y viceversa. Si un estudiante se ha presentado a uno de los dos exámenes, la nota que obtuvo en el mismo se está usando en el cálculo de la nota media de ese examen.

Las notas de entrada del grupo virtual son más altas que las del grupo presencial, esto significa que los estudiantes del grupo virtual ya partían con una mejor base que los del grupo presencial. Para hacer un análisis más objetivo de este conjunto de datos, vamos a cuantificar "el incremento de la nota media" en los dos grupos presencial y virtual.

En el grupo presencial este incremento fue de: 2.3333, mientras que en el grupo virtual fue de: 1.8125. Se evidencia que la media del incremento de la nota es más alta en el grupo presencial, pero ¿realmente la diferencia entre los dos grupos es significativa desde el punto de vista estadístico?

Preparamos los datos para comparar las medias de los dos grupos mediante la prueba no paramétrico de Wilcoxon, trabajando con el nivel de significación del $5 \%$, y se obtiene:

$\mathrm{W}=743$, $\mathrm{p}$-value $=0.6391$.

Dado que el p-valor mayor que todos los niveles de significación usuales $(0.01,0.05,0.10)$, se acepta la igualdad de las cantidades comparadas. Esto nos confirma que el nivel de aprendizaje que alcanzan los estudiantes que reciben el curso de Física Mecánica I en la modalidad virtual es similar al que alcanzan los que estudian de forma presencial, específicamente en el tema de Cinemática, tal y como habíamos comentado anteriormente.

Otro conjunto de resultados interesantes, son los que se obtuvieron al aplicar un instrumento (Tabla \# 6 del apéndice) que recopila un conjunto de criterios que permiten evaluar la nueva versión del curso de Física Virtual I. La gráfica se presenta a continuación:

\section{Gráfico 10.}

Criterios de los estudiantes.

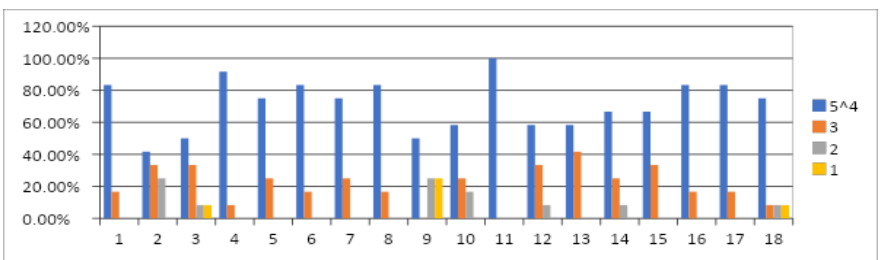

Nota. Criterio de los estudiantes, elaboración propia.

El instrumento cuenta de 18 ítems, y se le orientó a los estudiantes que cada una de las afirmaciones del cuestionario describe características que debe poseer un curso virtual, debe marcar con una «X» la casilla correspondiente según la frecuencia o intensidad con la que se cumple esa afirmación en el curso virtual. Respondiendo de acuerdo con la siguiente escala: 1: Nada, 2: Muy poco, 3: Algo, 4: Bastante, 5: Mucho.

El análisis de estos resultados nos lleva a detectar que más del $80 \%$ de los estudiantes evalúan muy bien ( 5 o 4) el nivel de comprensión de los contenidos, el uso de la bibliografía recomendada, 
el hecho de visitar al menos 2 veces por semana el Aula Virtual y la realización de las actividades asignadas. Sin embargo, reconocen que no utilizaron lo suficiente los medios de comunicación que ofrece el aula para canalizar sus dudas, y que no dedicaron tiempo suficiente al estudio. Estos son los ítems que se enfocan en lo que debe hacer el estudiante como individuo, que van desde el 1 al 10.

Los ítems del 11 al 18, son los que recogen criterios referidos al curso en cuestión. Estos datos nos llaman la atención sobre la buena evaluación que reciben:

1. A través de los distintos medios que ofrece el curso se potencia el pensamiento divergente, la discusión y el debate: Los docentes estimulan al alumno a hacer preguntas, reflexionar y a buscar respuestas.

2. La navegación del curso virtual es sencilla: facilita el desplazamiento y la localización de los recursos.

3. Diseño del curso virtual se caracteriza por presentar una apariencia visual agradable, equilibrada (imagen-texto, calidad-tamaño de imágenes), ser dinámico e innovador y facilitar el estudio.

Esto pone en evidencia que ha habido una mejoría notable en la nueva versión del Curso Virtual, su estructura, su facilidad para navegar, así como en la manera en que se presentan los contenidos. No obstante, más del $40 \%$ de los estudiantes evaluaron con nota regular:

- El curso virtual presenta exactitud y claridad de los contenidos: Los contenidos didácticos son precisos, fiables y objetivos, además de presentarse de forma comprensible.

Estos resultados nos ponen en alerta acerca de la estructura didáctica del Curso. Por otro lado, vemos un $25 \%$ de los participantes, aproximadamente, dejando explicitado que no tomarían de nuevo un curso virtual. Son elementos que nos indican sobre qué direcciones debemos trabajar para mejorar la próxima versión del curso de Física Virtual I en todos los sentidos.

\section{Discusión}

Los resultados obtenidos muestran que la modalidad virtual del curso Física Mecánica ofrece resultados similares en el desempeño estudiantil que las tradicionales modalidades presenciales, al juzgar mediante el uso de exámenes estandarizados. Sin embargo, los resultados de las encuestas donde se pregunta percepción sobre el curso virtual se observan que, preferían trabajar la física en modalidad presencial, aun siendo una generación tradicionalmente clasificada como tecnológica. ¿Qué ventaja, en cuanto a modalidad siguen percibiendo estos estudiantes, tiene la enseñanza presencial? ¿De qué manera puede promoverse un currículo virtual que cumpla con las expectativas tanto del estudiantado como de los administradores universitarios?

Con la intención de atender esta falta de interés por cursos virtuales se ha diseñado una estrategia de diseño de OVAt la cual pretende hacer uso del criterio heurístico de lo estético como motivador extrínseco del aprendizaje de contenidos relevantes a nivel universitario. Se trata de una apuesta a la sensibilidad humana, a los estímulos sensoriales producidos por la imagen, el sonido y la interactividad en un ambiente estructurado de forma deliberada a través de tecnologías de información.

Elementos constitutivos del OVAt, modelo creado por diseño instruccional transdisciplinario:

1. La unidad didáctica virtual, debe contener un video introductorio donde el facilitador presenta el área bajo estudio del OVAt con: bosquejo, objetivos específicos, conceptos o ideas centrales, diversas actividades a combinar y opciones para una evaluación auténtica. Se reseñan elementos de manera motivacional y optimista para crear un ambiente de estudio empático y relajado.

2. Bosquejo Interactivo del OVAt para navegación lineal o alternativa por selección del estudiante. Se proveen hojas de cotejo con equivalencias de actividades y avalúo único.

3. Objetivos específicos del OVAt centrados en el estudiante, desglosados por competencias: Tecnológicas, Informáticas, Disciplinares, Sociales, Emocionales.

4. Presentación interactiva de los contenidos disciplinares por parte del facilitador con 
Los Objetos Virtuales de Aprendizaje Transdisciplinarios, como Alternativa a la Problemática Enseñanza y Aprendizaje de la Física en la Modalidad Virtual. apoyo audiovisual y enlaces a portales de Internet de referencia. Presentación narrada.

5. En el caso específico de Física Mecánica, ejercicios de práctica para destrezas matemáticas, resolución de problemas conceptuales y numéricos con retroalimentación automatizada pintoresca y lúdica. Videos con ejemplos similares a los de práctica donde el facilitador demuestra los algoritmos de resolución.

6. Actividades de Estudio Independiente, tales como: Análisis de Lectura, Estudio de Casos, Laboratorios Virtuales, Visualizadores, Simuladores, Modelos Conceptuales, Juegos Educativos, Bases de Datos, Portales Interactivos. Estudio dirigido por Preguntas Guías o Hojas de Trabajo. Pruebas cortas de comprensión para autoevaluación disponibles. Todo esto se modera en espacios interactivos de intercambio social donde el estudiante recibe insignias con contenidos audio visual según sus aportaciones.

7. Referencias útiles serán incluidas como materiales de apoyo, tales como: lecturas con diversidad en su nivel de detalles, exámenes de ejemplo, solucionario de problemas clásicos, presentaciones de clases pasadas, enlaces a Internet, artículos arbitrados, entre otros.

8. Aportaciones transdisciplinarias enfatizan los componentes epistemológicos e históricos para contextualizar los fundamentos teóricos del área bajo estudio. Se aplicará el área bajo estudio en un contexto cotidiano con ejemplos de la vida real.

9. Evaluación Auténtica será fomentada durante toda la experiencia con el uso de diarios reflexivos y portafolios electrónicos para favorecer la metacognición y el auto-avalúo. Se ofrecerá al estudiante diversidad de herramientas equivalentes de avalúo para que construya su avalúo único combinando sus actividades con las herramientas de avalúo.

\section{Conclusiones}

Este estudio encontramos comportamientos similares, en cuanto a calificaciones obtenidas en pruebas estandarizadas, en el curso virtual de Física
Mecánica con su contraparte presencial. Sin embargo, otros aspectos relacionados al aprovechamiento estudiantil no quedan evaluados por el estudio.

Aspectos tales como nivel de envolvimiento, compromiso, sentido de pertenencia, entre otros no fueron medidos por el estudio y resultaría interesante considerarlos como líneas alternas de investigación sobre el éxito estudiantil en medios virtuales.

La enseñanza aprendizaje de física, tanto en entornos virtuales como presenciales sigue siendo un reto a nivel universitario. Debemos mantenernos atentos a toda alternativa que puedan generar actitudes positivas en los procesos de enseñanzaaprendizaje. Las tecnologías de información han demostrado ser medios valiosos de masificación de ofertas, tanto en entretenimiento como a nivel educativo, sin embargo, en este último debemos ser vigilantes en cuanto a la calidad de los ofrecimientos y el cumplimiento de expectativas.

Pensamos que los OVAt pueden ser una pieza clave en el desarrollo de materiales curriculares ajustados mejor a las necesidades de los procesos educativos actuales. Su diseño se fundamenta en una hermenéutica holística e integradora entre los contenidos relevantes y los aspectos psicológicos; estéticos, sociales, y motivacionales entre otros.

\section{Referencias}

Aceituno, J.A. (2014). "Algunas carencias detectadas en los estudiantes que ingresan a INTEC y reciben Física General en sus carreras. Consideraciones sobre el desarrollo y uso del video juego en la enseñanza de la Física". Ciencia y Sociedad. 39(4), 731-58.

Aceituno, J.A. Mujica, V.M. (2016) "Validación del ambiente virtual en tres dimensiones: La Mansión de La Física, como video juego para el aprendizaje". Ciencia y Sociedad. 41(4), 869-02.

Adams, W. K. (2010). "Student Engagement and Learning with PhET Interactive Simulations, Multimedia in Physics Teaching and Learning Proceedings". 2010. IL NUOVO CIMENTO. DOI 10.1393/ncc/i2010-10623-0

Adams, W. K.; Paulson, A. and Wieman, C. E. (2009) "What Levels of Guidance Promote Engaged Exploration with Interactive Simulations?" 2009. PERC Proceedings.

Arias, M.; Romero, V y Pérez, V. "Informe Física General I". 2011, INTEC.

Barráez, D. P. (2020). La educación a distancia en los procesos educativos: Contribuye significativamente al aprendizaje. Revista Tecnológica-Educativa Docentes 2.0, 8(1), 41-49. Recuperado de: https://ojs.docentes20.com/index.php/revistadocentes20/article/view/91 
Los Objetos Virtuales de Aprendizaje Transdisciplinarios, como Alternativa a la Problemática Enseñanza y Aprendizaje de la Física en la Modalidad Virtual.

Beichner, R. J. (1994). "Testing student interpretation of kinematics graph". Am. J. Phys.Vol.62, No.8

Christian, W.; Esquembre, F.; Barbato, L. (2011). "SPORE Award: Open Source Physics". http://www.compadre.org/portal/

Clements, P. \& Northrop, L. (2002). "Software Product Lines: Practices and Patterns". Addison-Wesley.

Escobar, M. (2017). A study of the way five teachers makes decisions in the "EFL Classroom". URI: http://dspace.uhemisferios.edu.ec:8080/xmlui/handle/1234567 89/944)

Esquembre, F.; Martin, E.; Christian, W.; Belloni, M. (2004). FISLEST: "Enseñanza de la Física con material interactivo". Capella, I. (edit); Hestenes, D. (prol); Madrid. Pearson Education, S. A. 456 p. ISBN: 84-205-3781-0

Filmer, D.; Hasan, A. y Pritchett, L. (2006). “A Millennium Learning Goal: Measuring Real Progress in Education - Working Paper 97". Center for Global Development.Hestenes, D.; Wells, M. and Swackhamer, G. (1992). Phys. Teach. 30, 141.

Gable, P. \& Harmon-Jones, E. (2010). The motivational dimensional model of affect: Implications for breadth of attention, memory, and cognitive categorization. Journal Cognition and Emotion 24(2), 322-337. https://doi.org/10.1080/02699930903378305

Lieury, A., Fenouillet, F. (2016) Motivación y éxito escolar Primera edición electrónica Fondo de cultura económica de Argentina SA Buenos Aires.

Mujica, R. (2019). Sociedad de la Información. Revista TecnológicaEducativa Docentes 2.0, 6(4), 7-8. Recuperado a partir de https://ojs.docentes20.com/index.php/revistadocentes20/article/view/74

Pintrich, P.R. (1999). The role of motivation in promoting and sustaining self-regulated learning. International Journal of Educational Research 31, 459-470. https://www.doi.org/10.12691/education-1-8-11

\section{Apéndice}

\begin{tabular}{|c|c|c|c|c|}
\hline ID & $\begin{array}{l}\text { Program } \\
\text { a }\end{array}$ & Nombre & Nota Final & $\alpha$ \\
\hline 1063731 & IND & ABREU MOLINA, DAVID ISRAEL & $\mathrm{R}$ & $\mathrm{R}$ \\
\hline 1067812 & SIS & BATISTA NUÑEZ, LUIS ANGEL & 64 & $\mathrm{D}$ \\
\hline 1065400 & ELE & BIDO CUELLO, EZEQUIAS & 71 & $\mathrm{C}$ \\
\hline 1069186 & MAT & $\begin{array}{l}\text { DE LA CRUZ ESPINOSA, } \\
\text { CATHERINE }\end{array}$ & 82 & B \\
\hline 1070262 & INQ & $\begin{array}{l}\text { DELGADO RAVELO, MARIA } \\
\text { VERONICA }\end{array}$ & 70 & $\mathrm{C}$ \\
\hline 1061434 & MAT & $\begin{array}{l}\text { DOMINGUEZ MELO KARLA } \\
\text { VICTORIA }\end{array}$ & 91 & A \\
\hline 1067951 & IND & $\begin{array}{l}\text { ESTEVEZ PAULINO, EDUARD } \\
\text { SAMUEL }\end{array}$ & 60 & $\mathrm{D}$ \\
\hline 1061763 & CIV & FUENZALINDA DURAN, JULIO A. & 60 & $\mathrm{D}$ \\
\hline 1071069 & IMC & GARCIA CASTILLO, GIANLUIS & $\mathrm{R}$ & $\mathrm{R}$ \\
\hline 1065785 & INL & GUZMAN MERCEDES, FELIX E. & 75 & $\mathrm{C}$ \\
\hline 1066209 & IND & $\begin{array}{l}\text { HOLGUIN MONTERO, LAURA } \\
\text { LETICIA }\end{array}$ & $\mathrm{R}$ & $\mathrm{R}$ \\
\hline 1065779 & IND & MATOS DIAZ, HERODES & 80 & B \\
\hline 1070162 & IEE & MELO TORIBIO, EDWARD A. & $\mathrm{R}$ & $\mathrm{R}$ \\
\hline 1062672 & IMC & MENDEZ GONZALEZ, JUAN C. & $\mathrm{R}$ & $\mathrm{R}$ \\
\hline 1067244 & IND & PEÑA MELO, JULIO RADHAMES & 60 & $\mathrm{D}$ \\
\hline 1066845 & IDS & $\begin{array}{l}\text { PRADEL FERMIN, LUCAS } \\
\text { ERNESTO }\end{array}$ & 80 & B \\
\hline 1068011 & CIV & RAMOS SANCHEZ, JOBERT C. & $\mathrm{R}$ & $\mathrm{R}$ \\
\hline 1067443 & CIV & SOLER CLETO, DARYS ANTONIO & 60 & $\mathrm{D}$ \\
\hline 1066679 & IND & $\begin{array}{l}\text { TAVAREZ VIDAL, MIGUEL } \\
\text { ARISTIDES }\end{array}$ & 63 & $\mathrm{D}$ \\
\hline 1068622 & CIV & $\begin{array}{l}\text { YASUOKA MONTAS, NAMI } \\
\text { ALTAGRACIA }\end{array}$ & 70 & $\mathrm{C}$ \\
\hline 1069136 & IMC & ACOSTA ACOSTA, CARLOS JESUS & 77 & $\mathrm{C}$ \\
\hline 1070229 & IDS & ARIAS MORILLO, JOSE RAMON & $\mathrm{R}$ & $\mathrm{R}$ \\
\hline 1070044 & CIV & $\begin{array}{l}\text { BERAS CARABALO, JULIO } \\
\text { ENRIQUE }\end{array}$ & $\mathrm{R}$ & $\mathrm{R}$ \\
\hline 1070947 & CIV & BURGOS BAUTISTA, ANGEL A. & 76 & $\mathrm{C}$ \\
\hline 1071055 & IND & CARRASCO GUZMAN, GENESIS & 70 & $\mathrm{C}$ \\
\hline 1069585 & IMC & DE LA MOTA YEARA, BRANDON & 70 & $\mathrm{C}$ \\
\hline 1069731 & IND & DIAS HERNANDEZ BREWICHS & $\mathrm{R}$ & $\mathrm{R}$ \\
\hline 1067862 & SIS & $\begin{array}{l}\text { GIBERTONI HERNANDEZ, } \\
\text { RAMSES J. }\end{array}$ & 60 & $\mathrm{D}$ \\
\hline 1069813 & IND & GONZALEZ RODRIGUEZ, JUAN E. & 80 & B \\
\hline 1066980 & IND & $\begin{array}{l}\text { GURIDI GUZMAN, VINKGRID } \\
\text { ANN }\end{array}$ & 70 & $\mathrm{C}$ \\
\hline 1070007 & CIV & GUTIERREZ SANTOS, MARIO & 61 & $\mathrm{D}$ \\
\hline 1070959 & SIS & $\begin{array}{l}\text { HERNANDEZ PEREZ, FERNANDO } \\
\text { E. }\end{array}$ & 80 & B \\
\hline 1071659 & IDS & LLUBERES MEJIA, YASSER A. & $\mathrm{R}$ & $\mathrm{R}$ \\
\hline 1069541 & IDS & MARION LANDAIS, LEANTHONY & $\mathrm{R}$ & $\mathrm{R}$ \\
\hline 1071504 & IND & MIESES MARTINEZ, JERY DANIA & $\mathrm{R}$ & $\mathrm{R}$ \\
\hline 1070224 & IND & NÚÑEZ FAMILIA, EDDY SAMUEL & 63 & $\mathrm{D}$ \\
\hline 1070541 & IMC & QUIÑONEZ QUESADA, DANIEL A. & 80 & B \\
\hline 1070282 & CIV & $\begin{array}{l}\text { RIVAS GUERRA, DANIEL } \\
\text { ENRIQUE }\end{array}$ & 83 & B \\
\hline 1069859 & SIS & $\begin{array}{l}\text { SILVESTRE DE JESUS, ADRIAN } \\
\text { ARTURO }\end{array}$ & 81 & B \\
\hline 1069239 & CIV & YI PEGUERO, LUIS FELIPE & 72 & $\mathrm{C}$ \\
\hline
\end{tabular}


Los Objetos Virtuales de Aprendizaje

Transdisciplinarios, como Alternativa a la Problemática Enseñanza y Aprendizaje de la Física en la Modalidad Virtual.

\section{Tabla 2}

Criterio de los estudiantes.

\begin{tabular}{|c|c|c|c|}
\hline Est & Positivo & Negativo & Sugerencias \\
\hline \multirow[t]{2}{*}{1} & $\begin{array}{l}\text { Acceso al material de } \\
\text { estudio siempre que lo } \\
\text { necesite }\end{array}$ & $\begin{array}{l}\text { Muy poco material de video } \\
\text { en las primeras unidades }\end{array}$ & \multirow[t]{2}{*}{$\begin{array}{l}\text { Más horas de } \\
\text { monitoria } \\
\text { presencial }\end{array}$} \\
\hline & $\begin{array}{l}\text { Flexibilidad en el } \\
\text { tiempo en que uno } \\
\text { puede estudiar. }\end{array}$ & $\begin{array}{l}\text { Ejemplos muy teóricos. (Se } \\
\text { necesitan más problemas } \\
\text { resueltos) }\end{array}$ & \\
\hline \multirow[t]{2}{*}{2} & $\begin{array}{l}\text { Aprendí a estudiar de } \\
\text { manera independiente }\end{array}$ & Deficiencia Técnica & $\begin{array}{l}\text { Mejoraría la } \\
\text { técnica }\end{array}$ \\
\hline & $\begin{array}{l}\text { Reforcé mi valor de } \\
\text { trabajo en equipo }\end{array}$ & $\begin{array}{l}\text { Sistema Educativo no nos } \\
\text { prepara para estudiar } \\
\text { independiente }\end{array}$ & $\begin{array}{l}\text { En las tutorías } \\
\text { presenciales, en } \\
\text { vez de responder } \\
\text { las dudas, debería } \\
\text { abordarse el tema } \\
\text { en cuestión. }\end{array}$ \\
\hline \multirow[t]{4}{*}{3} & \multirow[t]{4}{*}{$\begin{array}{l}\text { Motiva al estudiante a } \\
\text { crear hábito de estudio }\end{array}$} & $\begin{array}{l}\text { Poca interacción con el } \\
\text { profesor }\end{array}$ & $\begin{array}{l}\text { Enseñar la materia } \\
\text { de forma } \\
\text { presencial }\end{array}$ \\
\hline & & $\begin{array}{l}\text { Pocos videos de ejemplos } \\
\text { resueltos de forma detallada }\end{array}$ & $\begin{array}{l}\text { Subir más videos } \\
\text { de ejemplos } \\
\text { resueltos de forma } \\
\text { detallada. }\end{array}$ \\
\hline & & \multirow[t]{2}{*}{$\begin{array}{l}\text { Pocos puntos en los } \\
\text { exámenes (preguntas) }\end{array}$} & $\begin{array}{l}\text { Más horas de } \\
\text { tutorías } \\
\text { presenciales. }\end{array}$ \\
\hline & & & $\begin{array}{l}\text { Poner un examen } \\
\text { con más preguntas. }\end{array}$ \\
\hline \multirow[t]{3}{*}{5} & $\begin{array}{l}\text { Control del tiempo } \\
\text { (independencia) }\end{array}$ & $\begin{array}{l}\text { Problemas con la } \\
\text { plataforma, a veces los } \\
\text { videos no corren y algunos } \\
\text { símbolos no aparecen }\end{array}$ & $\begin{array}{l}\text { Más elementos } \\
\text { audiovisuales }\end{array}$ \\
\hline & $\begin{array}{l}\text { Acceso al material de } \\
\text { estudio en cualquier } \\
\text { momento }\end{array}$ & $\begin{array}{l}\text { Algunas dudas son difíciles } \\
\text { de resolver virtualmente, es } \\
\text { mejor en persona. }\end{array}$ & \multirow{2}{*}{$\begin{array}{l}\text { La tutoría } \\
\text { presencial debe ser } \\
\text { en horario de } \\
\text { clases, para que } \\
\text { todos puedan } \\
\text { asistir }\end{array}$} \\
\hline & $\begin{array}{l}\text { Se pueden hacer } \\
\text { preguntas en el foro en } \\
\text { el momento en que } \\
\text { surjan. }\end{array}$ & $\begin{array}{l}\text { Algunos aspectos de la } \\
\text { Teoría deberían explicarse } \\
\text { presencialmente, pues son } \\
\text { muy complicados }\end{array}$ & \\
\hline 6 & $\begin{array}{l}\text { Materiales siempre } \\
\text { disponibles }\end{array}$ & $\begin{array}{l}\text { Pocas horas de tutoría } \\
\text { presencial }\end{array}$ & $\begin{array}{l}\text { Aumentaría las } \\
\text { horas de tutoría } \\
\text { presencial }\end{array}$ \\
\hline 7 & $\begin{array}{l}\text { Que puedes dedicar el } \\
\text { tiempo que quieras y } \\
\text { no pierdes tiempo } \\
\text { viniendo al aula }\end{array}$ & $\begin{array}{l}\text { Que sin la presencia del } \\
\text { profesor es muy difícil } \\
\text { entender los temas. }\end{array}$ & \\
\hline 8 & $\begin{array}{l}\text { Poder estudiar desde } \\
\text { cualquier lugar }\end{array}$ & Explicar mejor los temas & \\
\hline 9 & $\begin{array}{l}\text { Ayuda a crear hábito } \\
\text { de estudio }\end{array}$ & $\begin{array}{l}\text { No vemos al profesor } \\
\text { explicando un tema como } \\
\text { tal, y puede confundir a la } \\
\text { hora de las medidas o } \\
\text { fórmulas. }\end{array}$ & $\begin{array}{l}\text { Más videos } \\
\text { explicando los } \\
\text { temas. }\end{array}$ \\
\hline 8 & $\begin{array}{l}\text { Se aprende a } \\
\text { investigar, porque } \\
\text { cuando no se entiende } \\
\text { algo se lee el libro o se } \\
\text { busca en internet, en } \\
\text { vez de hacer que el } \\
\text { profesor repita las } \\
\text { explicaciones varias } \\
\text { veces }\end{array}$ & Explicar mejor los temas & $\begin{array}{l}\text { La hora de la } \\
\text { monitoria } \\
\text { presencial que } \\
\text { coincida con la } \\
\text { hora de clase }\end{array}$ \\
\hline \multirow[t]{2}{*}{10} & $\begin{array}{l}\text { Se le está dando un } \\
\text { uso más productivo a } \\
\text { la tecnología. }\end{array}$ & \multirow[t]{2}{*}{$\begin{array}{l}\text { Física no es una materia que } \\
\text { se debería dar virtual, ya que } \\
\text { se basa mucho en la } \\
\text { práctica, y no me parece que } \\
\text { sea muy fácil implementarla } \\
\text { virtualmente. } \\
\text { Algunos estudiantes } \\
\text { necesitan ver a un profesor } \\
\text { al frente explicándoles para } \\
\text { poder entender. }\end{array}$} & $\begin{array}{l}\text { Ninguno, porque } \\
\text { no creo que se } \\
\text { deba impartir de } \\
\text { esta forma }\end{array}$ \\
\hline & $\begin{array}{l}\text { Ayuda la estudiante a } \\
\text { que sea independiente } \\
\text { del profesor }\end{array}$ & & $\begin{array}{l}\text { La hora de la } \\
\text { monitoria } \\
\text { presencial que } \\
\text { coincida con la } \\
\text { hora de clase }\end{array}$ \\
\hline
\end{tabular}

\begin{tabular}{|c|c|c|c|}
\hline 11 & $\begin{array}{l}\text { Elijo yo el momento de } \\
\text { trabajar. }\end{array}$ & $\begin{array}{l}\text { Los videos a veces } \\
\text { daban error y o se } \\
\text { visualizaban }\end{array}$ & $\begin{array}{l}\text { Poner más } \\
\text { ejemplos } \\
\text { explicados en } \\
\text { videos }\end{array}$ \\
\hline 12 & $\begin{array}{l}\text { Interiorizo más los temas } \\
\text { cuando los aprendo } \\
\text { independientemente, que } \\
\text { cuando me lo explican }\end{array}$ & $\begin{array}{l}\text { Algunos estudiantes } \\
\text { necesitan ver a un } \\
\text { profesor al frente } \\
\text { explicándoles para } \\
\text { poder entender. }\end{array}$ & $\begin{array}{l}\text { Más videos } \\
\text { explicando los } \\
\text { temas. }\end{array}$ \\
\hline 13 & Excelente maestro & $\begin{array}{l}\text { La selección debió ser } \\
\text { virtual } \\
\text { El sistema puede } \\
\text { mejorar }\end{array}$ & \\
\hline \multirow[t]{2}{*}{14} & $\begin{array}{l}\text { Se le está dando un uso } \\
\text { más productivo a la } \\
\text { tecnología. }\end{array}$ & \multirow[t]{2}{*}{$\begin{array}{l}\text { El sistema puede } \\
\text { mejorar } \\
\text { En la selección no se } \\
\text { informó que era una } \\
\text { asignatura virtual. }\end{array}$} & $\begin{array}{l}\text { Ninguno, porque } \\
\text { no creo que se } \\
\text { deba impartir de } \\
\text { esta forma }\end{array}$ \\
\hline & $\begin{array}{l}\text { Ayuda a que seamos más } \\
\text { independientes y } \\
\text { responsables a la hora de } \\
\text { estudiar. }\end{array}$ & & $\begin{array}{l}\text { Más videos de } \\
\text { ejemplos } \\
\text { resueltos }\end{array}$ \\
\hline \multirow[t]{2}{*}{15} & $\begin{array}{l}\text { Siempre las clases a } \\
\text { tiempo. }\end{array}$ & & \multirow{2}{*}{$\begin{array}{l}\text { Hay que } \\
\text { informar que la } \\
\text { asignatura es } \\
\text { virtual en la } \\
\text { preselección y la } \\
\text { selección }\end{array}$} \\
\hline & $\begin{array}{l}\text { Ayuda y aclaración de } \\
\text { dudas en cualquier } \\
\text { momento }\end{array}$ & $\begin{array}{l}\text { Algunos estudiantes } \\
\text { necesitan ver a un } \\
\text { profesor al frente } \\
\text { explicándoles para } \\
\text { poder entender. }\end{array}$ & \\
\hline \multirow[t]{3}{*}{14} & $\begin{array}{l}\text { Novedoso, utilización de } \\
\text { la Tecnología }\end{array}$ & \multirow{3}{*}{$\begin{array}{l}\text { Poca costumbre de } \\
\text { tomar materias en la } \\
\text { modalidad virtual }\end{array}$} & \multirow{3}{*}{$\begin{array}{l}\text { Al aula ninguno. } \\
\text { A nosotros los } \\
\text { estudiantes, que } \\
\text { debemos } \\
\text { entender que } \\
\text { estamos } \\
\text { entrando a una } \\
\text { nueva era }\end{array}$} \\
\hline & $\begin{array}{l}\text { Creo espíritu de } \\
\text { autocontrol y } \\
\text { responsabilidad. }\end{array}$ & & \\
\hline & Comodidad & & \\
\hline \multirow[t]{3}{*}{15} & Horario flexible & $\begin{array}{l}\text { Menos exclusividad } \\
\text { para las clases }\end{array}$ & \multirow{3}{*}{$\begin{array}{l}\text { Videoconferenci } \\
\text { as para aclarar } \\
\text { dudas en horario } \\
\text { de clases }\end{array}$} \\
\hline & \multirow[t]{2}{*}{ Menos presión } & $\begin{array}{l}\text { Poco tiempo de tutoría } \\
\text { presencial }\end{array}$ & \\
\hline & & $\begin{array}{l}\text { Mayor dificultad para } \\
\text { los exámenes por } \\
\text { lagunas }\end{array}$ & \\
\hline 16 & Puede manejar su tiempo & $\begin{array}{l}\text { Es una asignatura muy } \\
\text { complicada para } \\
\text { impartirla virtual }\end{array}$ & $\begin{array}{l}\text { Impartirla } \\
\text { semipresencial }\end{array}$ \\
\hline 17 & $\begin{array}{l}\text { Puede aprender } \\
\text { independientemente }\end{array}$ & & $\begin{array}{l}\text { Debe haber una } \\
\text { sección de } \\
\text { respuestas para } \\
\text { que alumno } \\
\text { pueda tener } \\
\text { retroalimentació } \\
\mathrm{n} \text { de los } \\
\text { ejercicios que } \\
\text { hace de práctica }\end{array}$ \\
\hline \multirow[t]{2}{*}{18} & $\begin{array}{l}\text { Muy práctico para } \\
\text { mejorar su propio tiempo }\end{array}$ & $\begin{array}{l}\text { No se entienden bien } \\
\text { los temas por falta de } \\
\text { tutoría presencial. }\end{array}$ & \multirow[t]{2}{*}{$\begin{array}{l}\text { horas } \\
\text { presenciales }\end{array}$} \\
\hline & $\begin{array}{l}\text { Ayuda a esforzarse para } \\
\text { estudiar por tu propia } \\
\text { cuenta. }\end{array}$ & $\begin{array}{l}\text { No hay suficientes } \\
\text { videos explicando los } \\
\text { conceptos básicos }\end{array}$ & \\
\hline \multirow[t]{2}{*}{19} & $\begin{array}{l}\text { Es cómodo a la hora de } \\
\text { estudiar }\end{array}$ & & \multirow[t]{2}{*}{$\begin{array}{l}\text { Incorporar más } \\
\text { videos }\end{array}$} \\
\hline & $\begin{array}{l}\text { Las tutorías presenciales } \\
\text { sirven para aclarar las } \\
\text { dudas }\end{array}$ & & \\
\hline \multirow[t]{5}{*}{20} & $\begin{array}{l}\text { Muy buen profesor, los } \\
\text { videos muy detallados }\end{array}$ & Poco tiempo presencial & \multirow[t]{2}{*}{ Tiempo } \\
\hline & Manejo del tiempo & $\begin{array}{l}\text { Es una materia muy } \\
\text { importante para darse } \\
\text { virtual }\end{array}$ & \\
\hline & & $\begin{array}{l}\text { Debió avisarse en la } \\
\text { selección. }\end{array}$ & Organización \\
\hline & & \multirow{2}{*}{$\begin{array}{l}\text { No había tanto contacto } \\
\text { con el profesor }\end{array}$} & Material dado \\
\hline & & & Tarea \\
\hline
\end{tabular}


Los Objetos Virtuales de Aprendizaje Transdisciplinarios, como Alternativa a la Problemática Enseñanza y Aprendizaje de la Física en la Modalidad Virtual.

\begin{tabular}{|c|c|c|c|}
\hline \multirow[t]{3}{*}{21} & $\begin{array}{l}\text { Se puede jugar con } \\
\text { nuestro tiempo }\end{array}$ & $\begin{array}{l}\text { Es más difícil entender los } \\
\text { contenidos sin verlos } \\
\text { explicados físicamente. }\end{array}$ & \\
\hline & $\begin{array}{l}\text { Facilidad de tener } \\
\text { acceso a los } \\
\text { contenidos }\end{array}$ & $\begin{array}{l}\text { Es una materia muy } \\
\text { demandante para darla } \\
\text { virtual. }\end{array}$ & \\
\hline & $\begin{array}{l}\text { Ahorro de gasolina y } \\
\text { tiempo }\end{array}$ & $\begin{array}{l}\text { El aula virtual es muy } \\
\text { defectuosa. }\end{array}$ & \\
\hline \multirow[t]{2}{*}{22} & \multirow[t]{2}{*}{$\begin{array}{l}\text { Organizas más tu } \\
\text { tiempo }\end{array}$} & $\begin{array}{l}\text { No puedes hacer preguntas } \\
\text { al instante }\end{array}$ & $\begin{array}{l}\text { No considero que } \\
\text { Física debería darse } \\
\text { virtual. }\end{array}$ \\
\hline & & $\begin{array}{l}\text { Difícil de comprender sin } \\
\text { alguien que te explique. }\end{array}$ & $\begin{array}{l}\text { En la selección } \\
\text { debería aparecer } \\
\text { que es virtual }\end{array}$ \\
\hline \multirow[t]{2}{*}{23} & \multirow[t]{2}{*}{ Videos explicativos } & $\begin{array}{l}\text { La forma de aprender no es } \\
\text { correcta }\end{array}$ & $\begin{array}{l}\text { Más presencia del } \\
\text { profesor }\end{array}$ \\
\hline & & $\begin{array}{l}\text { Sin la presencia del profesor } \\
\text { no me sentía cómodo. }\end{array}$ & $\begin{array}{l}\text { La materia virtual } \\
\text { no es tan efectiva }\end{array}$ \\
\hline \multirow[t]{2}{*}{24} & \multirow{2}{*}{$\begin{array}{l}\text { La monitoria } \\
\text { presencial eran } \\
\text { excelentes porque las } \\
\text { dudas las aclaraba el } \\
\text { profesor de manera } \\
\text { práctica y sencilla. }\end{array}$} & $\begin{array}{l}\text { Sin la presencia del profesor } \\
\text { no me sentía cómodo. }\end{array}$ & $\begin{array}{l}\text { La materia virtual } \\
\text { no es tan efectiva }\end{array}$ \\
\hline & & $\begin{array}{l}\text { Todo estudiante necesita una } \\
\text { motivación y personalmente } \\
\text { el ver al profesor aclarando } \\
\text { todas tus dudas varias veces } \\
\text { a la semana es esencial para } \\
\text { el aprendizaje y la } \\
\text { motivación que uno necesita }\end{array}$ & $\begin{array}{l}\text { Avisar al estudiante } \\
\text { que la clase será } \\
\text { virtual. }\end{array}$ \\
\hline
\end{tabular}

Nota. Elaboración propia.

\section{Tabla 4}

Entrada y Salida Presencial Nov-Ene 2019

\begin{tabular}{|c|c|c|}
\hline Contador & Entrada & Salida \\
\hline 1 & 11 & 1 \\
\hline 2 & 2 & 3 \\
\hline 3 & 8 & 13 \\
\hline 4 & 9 & 9 \\
\hline 5 & 5 & 7 \\
\hline 6 & 6 & 5 \\
\hline 7 & 8 & 9 \\
\hline 8 & 4 & 11 \\
\hline 9 & 1 & 1 \\
\hline 10 & 3 & 9 \\
\hline 11 & 6 & 7 \\
\hline 12 & 11 & 12 \\
\hline 13 & 5 & 5 \\
\hline 14 & 12 & 14 \\
\hline 15 & 1 & 6 \\
\hline 16 & 6 & 7 \\
\hline 17 & 6 & 5 \\
\hline 18 & 4 & 6 \\
\hline 19 & 7 & 12 \\
\hline 20 & 1 & 8 \\
\hline 21 & 4 & 8 \\
\hline 22 & 3 & 6 \\
\hline 24 & 5 & 11 \\
\hline 25 & 1 & 8 \\
\hline 26 & 8 & 13 \\
\hline 28 & 7 & 3 \\
\hline 29 & 9 & 5 \\
\hline 30 & 7 & 0 \\
\hline 31 & 4 & 5 \\
\hline 32 & 8 & 7 \\
\hline 34 & 5 & 5 \\
\hline 35 & 4 & 5 \\
\hline 36 & 13 & 14 \\
\hline 37 & 5 & 4 \\
\hline 38 & 0 & 0 \\
\hline 39 & 2 & 1 \\
\hline 40 & 7 & 5 \\
\hline 41 & 4 & 0 \\
\hline 42 & 18 & 17 \\
\hline 43 & 8 & 11 \\
\hline 45 & 11 & 13 \\
\hline 46 & 11 & 14 \\
\hline 47 & 5 & 3 \\
\hline 48 & & 4 \\
\hline 49 & 18 & 16 \\
\hline 50 & 5 & 6 \\
\hline 51 & 1 & 17 \\
\hline 52 & 1 & 2 \\
\hline 53 & 2 & 7 \\
\hline 54 & 11 & 19 \\
\hline 55 & 6 & 9 \\
\hline 56 & 15 & 16 \\
\hline
\end{tabular}

Entrada y salida. INTEC 2018

\begin{tabular}{|c|c|c|c|c|c|c|}
\hline \multirow[t]{2}{*}{ Nombre } & \multicolumn{6}{|c|}{ Cinemática } \\
\hline & Ent. & E\% Total & Sal. & $\begin{array}{l}\text { S\% } \\
\text { Total }\end{array}$ & $\begin{array}{l}\text { Dif. } \\
\%\end{array}$ & $\begin{array}{l}\text { Dif. } \\
\text { Ptos }\end{array}$ \\
\hline $\begin{array}{l}\text { ALONZO BROBERG, } \\
\text { EDUARDO FELIPE }\end{array}$ & 11 & $52.38 \%$ & 10 & $\begin{array}{l}47.62 \\
\%\end{array}$ & $\begin{array}{l}- \\
4.76 \\
\%\end{array}$ & -1 \\
\hline $\begin{array}{l}\text { CARMEN DE LOS } \\
\text { SANTOS SANTANA, } \\
\text { VALERIE MARCELLES } \\
\text { DEL }\end{array}$ & 2 & $9.52 \%$ & 3 & $\begin{array}{l}14.29 \\
\%\end{array}$ & $\begin{array}{l}4.76 \\
\%\end{array}$ & 1 \\
\hline $\begin{array}{l}\text { CARRASCO ROMERO, } \\
\text { CARLOS LENIN }\end{array}$ & 8 & $38.10 \%$ & 13 & $\begin{array}{l}61.90 \\
\%\end{array}$ & $\begin{array}{l}23.8 \\
1 \%\end{array}$ & 5 \\
\hline $\begin{array}{l}\text { GOMEZ DIAZ, HENRY } \\
\text { ANDRES }\end{array}$ & 9 & $42.86 \%$ & 9 & $\begin{array}{l}42.86 \\
\%\end{array}$ & $\begin{array}{l}0.00 \\
\%\end{array}$ & 0 \\
\hline $\begin{array}{l}\text { JUSTO MONTALVO, } \\
\text { VASTHI PRISCILLA }\end{array}$ & 5 & $23.81 \%$ & 7 & $\begin{array}{l}33.33 \\
\%\end{array}$ & $\begin{array}{l}9.52 \\
\%\end{array}$ & 2 \\
\hline $\begin{array}{l}\text { MARTINEZ } \\
\text { ENCARNACION, } \\
\text { DIANA LORENA }\end{array}$ & 6 & $28.57 \%$ & 5 & $\begin{array}{l}23.81 \\
\%\end{array}$ & $\begin{array}{l}- \\
4.76 \\
\%\end{array}$ & -1 \\
\hline $\begin{array}{l}\text { MÉNDEZ SOLER, } \\
\text { DANIEL ALEXANDER }\end{array}$ & 8 & $38.10 \%$ & 9 & $\begin{array}{l}42.86 \\
\%\end{array}$ & $\begin{array}{l}4.76 \\
\%\end{array}$ & 1 \\
\hline $\begin{array}{l}\text { PEÑA BERROA, } \\
\text { ISRAEL JOSE }\end{array}$ & 4 & $19.05 \%$ & 11 & $\begin{array}{l}52.38 \\
\%\end{array}$ & $\begin{array}{l}33.3 \\
3 \%\end{array}$ & 7 \\
\hline $\begin{array}{l}\text { PERDOMO MENA, } \\
\text { SIMON EDUARDO }\end{array}$ & 10 & $47.62 \%$ & 10 & $\begin{array}{l}47.62 \\
\%\end{array}$ & $\begin{array}{l}0.00 \\
\%\end{array}$ & 0 \\
\hline $\begin{array}{l}\text { RAMIREZ MONTERO, } \\
\text { ELIANOR }\end{array}$ & 3 & $14.29 \%$ & 9 & $\begin{array}{l}42.86 \\
\%\end{array}$ & $\begin{array}{l}28.5 \\
7 \%\end{array}$ & 6 \\
\hline $\begin{array}{l}\text { REMIGIO POLANCO, } \\
\text { ROGER }\end{array}$ & 6 & $28.57 \%$ & 7 & $\begin{array}{l}33.33 \\
\%\end{array}$ & $\begin{array}{l}4.76 \\
\%\end{array}$ & 1 \\
\hline $\begin{array}{l}\text { SILVESTRE MEJIA, } \\
\text { JOSE ANTONIO }\end{array}$ & 11 & $52.38 \%$ & 12 & $\begin{array}{l}57.14 \\
\%\end{array}$ & $\begin{array}{l}4.76 \\
\%\end{array}$ & 1 \\
\hline $\begin{array}{l}\text { SOSA RODRÍGUEZ, } \\
\text { MIGUEL ANGEL }\end{array}$ & 5 & $23.81 \%$ & 5 & $\begin{array}{l}23.81 \\
\%\end{array}$ & $\begin{array}{l}0.00 \\
\%\end{array}$ & 0 \\
\hline $\begin{array}{l}\text { TEMPLE, KYNAN } \\
\text { O'CONNELL }\end{array}$ & 12 & $57.14 \%$ & 14 & $\begin{array}{l}66.67 \\
\%\end{array}$ & $\begin{array}{l}9.52 \\
\%\end{array}$ & 2 \\
\hline $\begin{array}{l}\text { TORRES JOSE, LUIS } \\
\text { GERARDO }\end{array}$ & 10 & $47.62 \%$ & 6 & $\begin{array}{l}28.57 \\
\%\end{array}$ & $\begin{array}{l}- \\
19.0 \\
5 \%\end{array}$ & -4 \\
\hline $\begin{array}{l}\text { TRINIDAD REYES, } \\
\text { DARVIS VLADIMIR }\end{array}$ & 6 & $28.57 \%$ & 7 & $\begin{array}{l}33.33 \\
\%\end{array}$ & $\begin{array}{l}4.76 \\
\%\end{array}$ & 1 \\
\hline $\begin{array}{l}\text { VOLQUEZ MERETTE, } \\
\text { AVRIL CLARETTE }\end{array}$ & 6 & $28.57 \%$ & 5 & $\begin{array}{l}23.81 \\
\%\end{array}$ & $\begin{array}{l}- \\
4.76 \\
\%\end{array}$ & -1 \\
\hline $\begin{array}{l}\text { MORBÁN GÓMEZ, } \\
\text { NELSON ADRIAN }\end{array}$ & 4 & $19.05 \%$ & 6 & $\begin{array}{l}28.57 \\
\%\end{array}$ & $\begin{array}{l}9.52 \\
\%\end{array}$ & 2 \\
\hline $\begin{array}{l}\text { PIANTINI ARBAJE, } \\
\text { MARINO JESUS }\end{array}$ & 7 & $33.33 \%$ & 12 & $\begin{array}{l}57.14 \\
\%\end{array}$ & $\begin{array}{l}23.8 \\
1 \%\end{array}$ & 5 \\
\hline $\begin{array}{l}\text { ROSADO, JEURY } \\
\text { DAVID }\end{array}$ & 10 & $47.62 \%$ & 8 & $\begin{array}{l}38.10 \\
\%\end{array}$ & $\begin{array}{l}- \\
9.52 \\
\%\end{array}$ & -2 \\
\hline $\begin{array}{l}\text { MEDOS CRUZ, LUIS } \\
\text { RAFAEL }\end{array}$ & 4 & $19.05 \%$ & 8 & $\begin{array}{l}38.10 \\
\%\end{array}$ & $\begin{array}{l}19.0 \\
5 \%\end{array}$ & 4 \\
\hline
\end{tabular}

Nota. Elaboración propia. 
Los Objetos Virtuales de Aprendizaje

Transdisciplinarios, como Alternativa a la Problemática Enseñanza y Aprendizaje de la Física en la Modalidad Virtual.

Tabla 5.

Entrada y Salida Virtual. Nov-Ene 2019

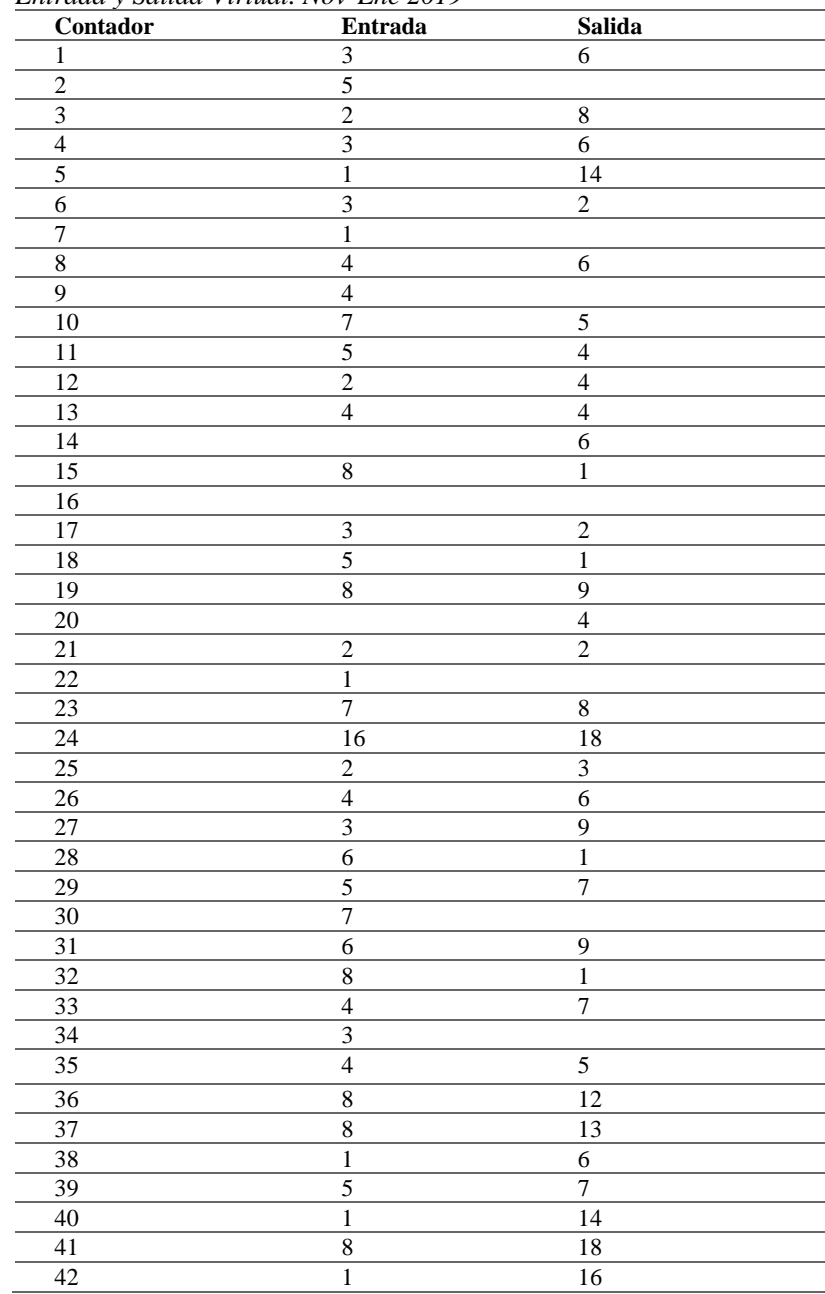

Nota. Elaboración propia.
Tabla 6.

Instrumento de recopilación de criterios

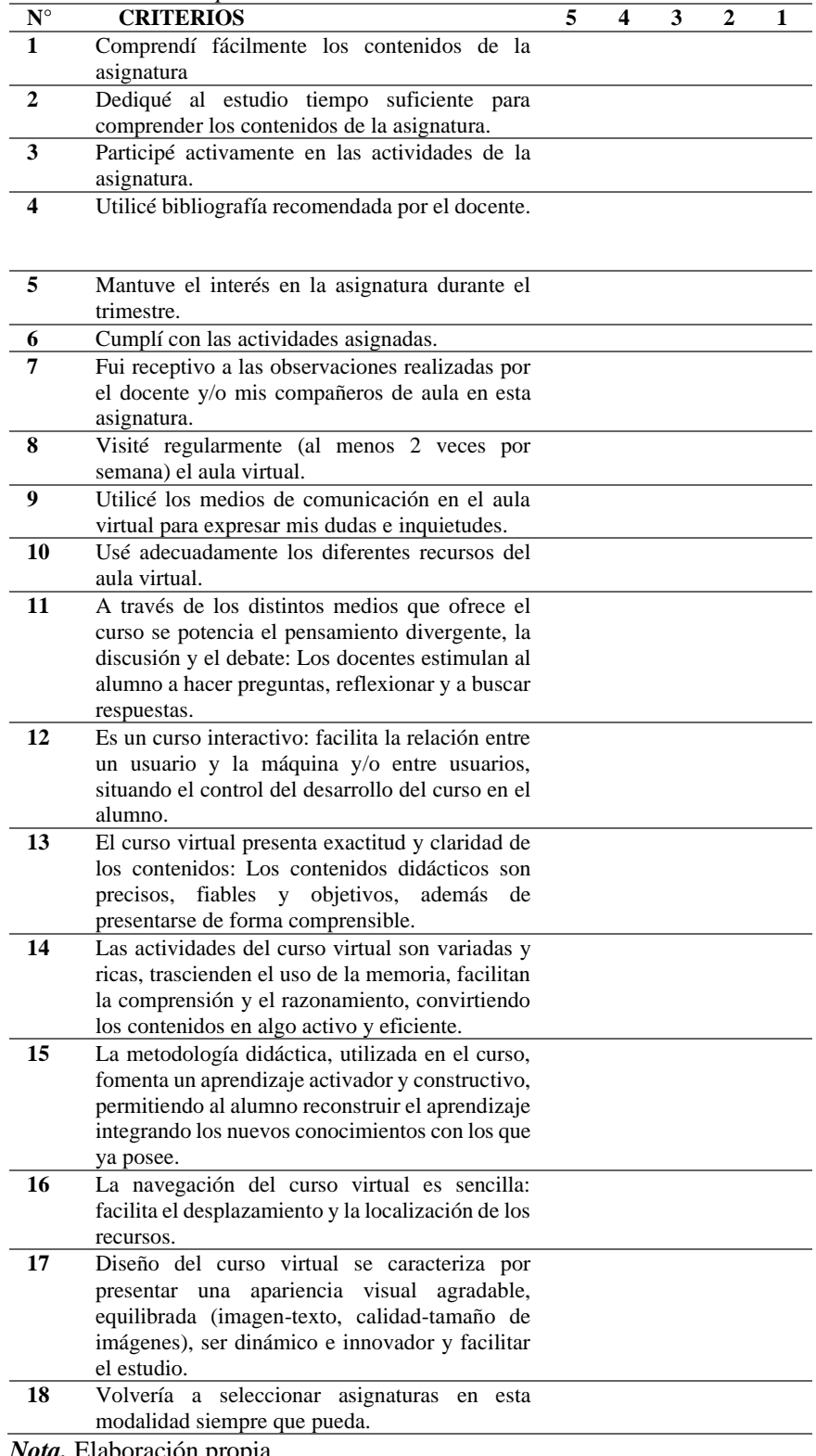

